\title{
The detection of a 1.4-h period in RW Ursa Minoris - candidate for shortest recorded orbital period nova
}

\author{
A. Retter ${ }^{1}$ and Y. Lipkin ${ }^{2}$ \\ 1 Dept. of Physics, Keele University, Keele, Staffordshire ST5 5BG, UK \\ 2 School of Physics and Astronomy and the Wise Observatory, Raymond and Beverly Sackler Faculty of Exact \\ Sciences, Tel-Aviv University, Tel Aviv 69978, Israel
}

Received 9 August 2000 / Accepted 19 October 2000

\begin{abstract}
CCD photometry of the classical nova RW UMi during 10 nights in 1995 through an $R$ filter and 14 nights in 1997 through a "clear" filter, with the 1-m Wise telescope yields a periodic modulation in the light curve with the period $0.05912 \pm 0.00015 \mathrm{~d}$ and a semi amplitude of 0.025 mag. We discuss several explanations for the periodicity, and suggest that it is likely to be the orbital period of the binary system or a superhump period. RW UMi is thus a candidate for the classical nova with the shortest known orbital period.
\end{abstract}

Key words. individual: RW UMi - novae, cataclysmic variables - binaries: close - accretion, accretion discs white dwarfs

\section{Introduction}

RW UMi is a high galactic latitude nova that erupted in 1956. Its outburst was reported, however, only about six years later (Kukarkin 1962). The nova reached a maximum of at least $m_{V} \approx 6.0$ on 1956 September 24 , and decayed to $m_{V} \approx 11.5$ a year later. Kukarkin also concluded that the progenitor of the nova was fainter than $m_{V}=21.2$ in POSS. Duerbeck (1987), however, barely detected the pre-nova at $m_{V}=21.0$ in the same plate. Photometric observations of the object during the last two decades are consistent with a visual magnitude stable around $m_{V} \approx 18.8$ (Cohen 1985; Kaluzny \& Chlebowski 1989; Szkody et al. 1989; Howell et al. 1991; Szkody \& Howell 1992; Szkody 1994; Ringwald et al. 1996; Downes \& Duerbeck 2000). We note that the estimate of the nova in 1988 as $m_{V}=21.0-21.5$ (Howell 1988) was corrected by Szkody et al. (1989).

Spectra of the post-nova are presented by Kaluzny \& Chlebowski (1989), Szkody \& Howell (1992) and Ringwald et al. (1996). These show a strong blue continuum and several weak emission lines - the Balmer and He II 4686 lines.

Cohen (1985) marginally detected a nebula around the remnant of RW UMi using an image taken through a narrow $\mathrm{H} \alpha$ filter. Slavin et al. (1995) repeated this observation, and concluded that the distance to the binary system is $5 \pm 2 \mathrm{Kpc}$. Esenoglu et al. (2000) resolved the shell of

Send offprint requests to: A. Retter, e-mail: ar@astro.keele.ac.uk
RW UMi in 1995 and derived a distance range for the nova of 4200-5840 pc. Downes \& Duerbeck (2000) failed, however, to detect the nova nebula in an observation carried out in 1998.

Kaluzny \& Chlebowski (1989) found that the nova was not variable at the $0.05 \mathrm{mag}$ level in the $B$ and $V$ filters in five images taken in 1985 and one in 1987. Szkody et al. (1989) reported the discovery of a variation of about $0.2 \mathrm{mag}$ in a 3-h observation in 1988 using a wide band red filter. They suggested a possible period of $117 \pm 5 \mathrm{~min}$. Shafter \& Campbell (1990) independently announced a similar detection. Howell et al. (1991) observed the nova with a $B$ filter for $2.1 \mathrm{~h}$ in 1989 , and proposed a period of $113 \pm 10 \mathrm{~min}$.

There are only three recorded classical novae with established orbital periods below the cataclysmic variables (CVs) period gap - CP Pup 1942, GQ Mus 1983 and V1974 Cyg 1992 (Diaz \& Bruch 1997; Ritter \& Kolb $1998^{1}$ ). This is compared with about one hundred and twenty other short orbital period systems. In comparison, above the gap there are more than thirty novae and about one hundred and thirty other systems. Thus the ratio of novae to all CVs below the gap is nearly ten times smaller than above the gap. An increase of the number of known novae below the period gap is, therefore, necessary for the understanding of nova cycles of short orbital period CVs. The possibility that the orbital period in RW UMi is below the gap lead us to add this nova to

\footnotetext{
1 Note that the dwarf novae WX Cet and TV Crv were misclassified as novae.
} 
Table 1. The observations time table

\begin{tabular}{|c|c|c|c|c|c|}
\hline $\begin{array}{c}\text { UT } \\
\text { Date }\end{array}$ & $\begin{array}{c}\text { Time of } \\
\text { Start } \\
\text { (HJD- } \\
2449000) \\
\end{array}$ & $\begin{array}{c}\text { Run } \\
\text { Time } \\
\text { (h) }\end{array}$ & $\begin{array}{c}\text { Number } \\
\text { of } \\
\text { frames }\end{array}$ & Filter & notes \\
\hline 110595 & 849.275 & 0.9 & $2,6,1$ & $R, V, I$ & \\
\hline 250595 & 863.259 & 2.9 & 17 & $R$ & \\
\hline 060695 & 875.265 & 3.2 & 20 & $R$ & \\
\hline 090695 & 878.254 & 1.3 & 8 & $R$ & \\
\hline 100695 & 879.247 & 1.2 & 8 & $R$ & \\
\hline 110695 & 880.238 & 1.6 & 10 & $R$ & \\
\hline 180695 & 887.245 & 7.7 & 33 & $R$ & gaps \\
\hline 200695 & 889.239 & 1.6 & 10 & $R$ & \\
\hline 210695 & 890.234 & 1.4 & 9 & $R$ & \\
\hline 020795 & 901.271 & 7.2 & 117 & $R$ & \\
\hline 030795 & 902.252 & 7.5 & 105 & $R$ & gap \\
\hline 130597 & 1582.235 & 7.9 & 78 & Clear & \\
\hline 140597 & 1583.308 & 5.2 & 48 & Clear & \\
\hline 100697 & 1610.251 & 7.6 & 86 & Clear & \\
\hline 110697 & 1611.251 & 7.6 & 80 & Clear & \\
\hline 120697 & 1612.253 & 7.6 & 85 & Clear & \\
\hline 270697 & 1627.253 & 7.5 & 65 & Clear & gaps \\
\hline 070897 & 1668.255 & 7.3 & 68 & Clear & \\
\hline 080897 & 1669.252 & 7.0 & 65 & Clear & \\
\hline 090897 & 1670.242 & 6.8 & 54 & Clear & \\
\hline 270897 & 1688.223 & 3.3 & 32 & Clear & \\
\hline 280897 & 1689.236 & 8.0 & 76 & Clear & \\
\hline 310897 & 1692.235 & 7.2 & 62 & Clear & \\
\hline 010997 & 1693.225 & 4.0 & 36 & Clear & \\
\hline 020997 & 1694.213 & 8.3 & 71 & Clear & \\
\hline 101097- & $1732-$ & & & & \\
\hline 270799 & 2387 & & 98 & $R$ & snapshots \\
\hline
\end{tabular}

our list of targets in an ongoing programme, maintained at the Wise Observatory, to search for periods in post-novae. Retter (1999) announced the detection of a 1.4-h periodicity in the light curve of RW UMi. In this work we elaborate that report, discuss possible physical mechanisms for the variation and further try to classify the system within the known subgroups of CVs.

\section{Observations}

We observed Nova RW UMi during 10 nights in 1995 through a standard Cousins $R$ filter and 14 nights in 1997 through a "clear" filter. A few images of the nova in the $V, R$ and $I$ filters were taken as well in 1995 May 11. In addition, one snapshot per night through the $R$ filter was taken on 98 occasions between 1997 October and 1999 July. Table 1 presents a summary of the observation schedule. The photometry was carried out using the 1-m telescope at the Wise Observatory, with the Tektronix $1 \mathrm{~K}$ CCD camera. The typical exposures times were 3-15 $\mathrm{m}$ in 1995 and 5-6 $\mathrm{m}$ in 1997-1999.

Bias and flat field corrections were made to each frame. Aperture photometric measurements were carried out using the DAOPHOT program (Stetson 1987). Differential magnitudes of the nova relative to several reference stars

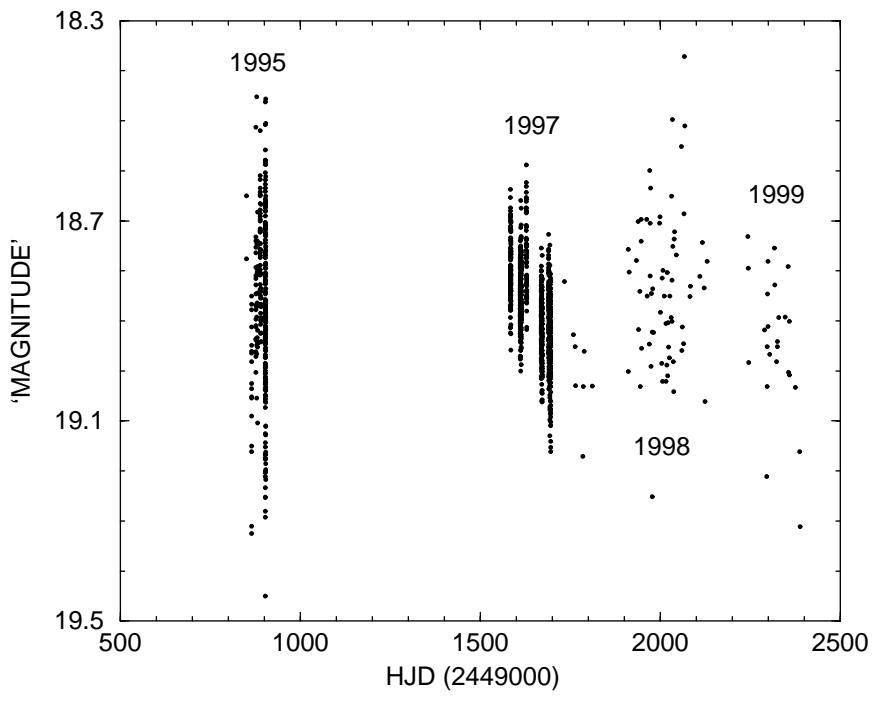

Fig. 1. All 1995-1999 data points are presented. Note that this is a combination of two filters ( $R$ and "clear"). In 1995 and 1997 RW UMi was observed for a few hours each night; from winter 1997 on only snapshots were taken. In 1997 the object faded by about $0.1 \mathrm{mag}$ during the second part of the observations (August-September) relative to the first part (May-June)

(the exact number depending on each image quality) were obtained from the images. The Wise Observatory reduction program DAOSTAT (Netzer et al. 1996) was used to reject variable comparison stars. The number of frames obtained in each filter on our programme is: $437(R), 906$ ("clear"), $6(V)$ and $1(I)$. The mean of the errors in the observations in the $R$ and "clear" filters were 0.11 and 0.04 mag correspondingly.

Figure 1 presents all observations in the $R$ and "clear" bands during 1995-1999. The two filters were combined assuming that the mean "clear" magnitude of the comparison stars is the same as the mean $R$ magnitude. The graph shows that the colour $R$-"clear" of the nova is relatively small compared with the scatter of the points that cannot be attributed only to the observational errors. The nightly means in 1997 varied by a few tenths mag from night to night with a standard deviation of $\sigma=0.09 \mathrm{mag}-$ more than twice the mean error of the data points. During the first six nights in 1997 the nova appeared to be consistently about $0.1 \mathrm{mag}$ brighter than the remaining eight nights.

\section{Data analysis}

The best nights in the 1995 data showed a possible variation of the order of 1-3 h, however, since an $R$ filter was used, the errors were much larger than the 1997 data when more photons were accumulated using a "clear" filter. In addition, the length of run on each night in 1995 was generally very short and two out of the three longest nights have gaps in between. Therefore, the power spectrum of these data is very noisy.

The 1997 data were obtained without a filter, thus having smaller errors. The mean length of the runs was $6.8 \mathrm{~h}$. 


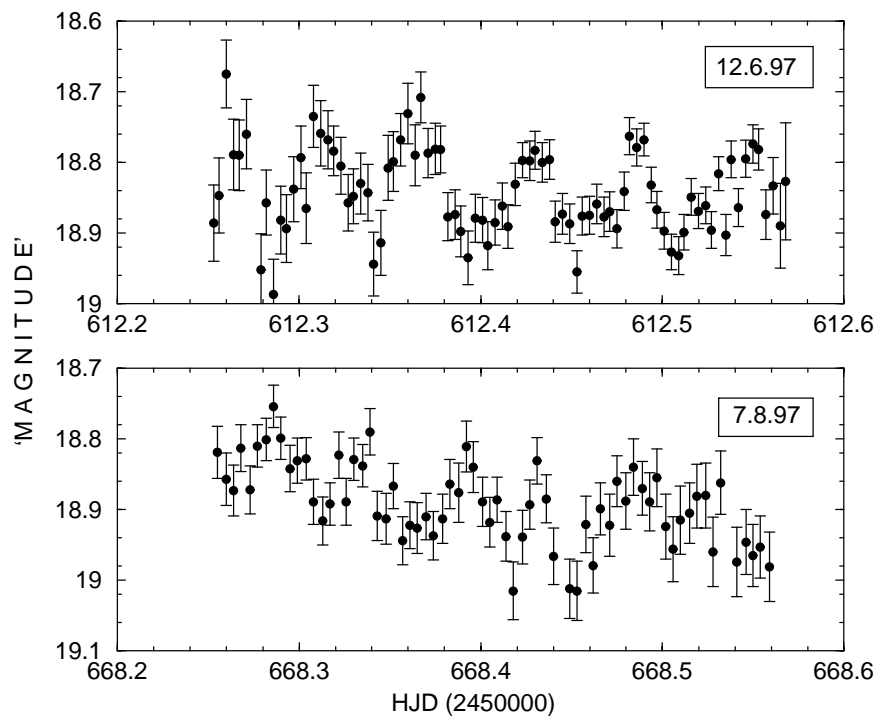

Fig. 2. Light curves of two of our best nights in 1997 obtained using a "clear" filter. The upper panel shows the points of June 12 and lower panel - August 7. More than five cycles of the 1.4-h periodicity can be seen in each night

A variation of $\sim 1.4 \mathrm{~h}$ with a full amplitude of $0.1-0.2 \mathrm{mag}$ can be seen by simple visual inspection of the light curve in a few of the best nights. Figure 2 presents two examples June 12 and August 7. In both light curves more than five cycles of the candidate periodicity are easily seen. Note the overall fading during the second night. Such gradients appeared in the light curves of several other nights as well.

The power spectrum of the 1997 data obtained through the "clear" filter is shown in Fig. 3. The detrending was carried out by subtracting the mean from each night. The highest peak at mid-frequencies $\left(f=16.916 \mathrm{~d}^{-1}\right)$ corresponds to a period of $0.05912 \mathrm{~d}$. It is many standard deviations above the noise level. Aliases of this peak are also present. To check whether the second highest peak at mid-frequencies after the $16.916 \mathrm{~d}^{-1}$ frequency, its 1-d alias at $17.916 \mathrm{~d}^{-1}$, is the correct period, we carried out the following test. First a synthetic light curve was built using a sinusoid at this period. This sinusoid was given the same amplitude it has in the data and sampled according to the window function. Next noise was added using the rms of the residuals of the fit to the data. We then checked whether the highest peak in a small interval around the $16.916 \mathrm{~d}^{-1}$ frequency is higher than the strongest peak near the $17.916 \mathrm{~d}^{-1}$ frequency. One thousand simulations show that the probability that the $1-\mathrm{d}$ alias at $17.916 \mathrm{~d}^{-1}$ $(0.05582 \mathrm{~d})$ is the correct period is $4 \%$.

The group of peaks at the left-hand side of the diagram is centered around the $2.0 \mathrm{~d}^{-1}$ peak that appears in the window function as well. Other low-frequency peaks correspond to periodicities that are longer than the typical interval of observations in each night, and probably reflect the strong noise level and/or gradients that appear



Fig. 3. The power spectrum of the 1997 points ("clear" filter) after the subtraction of the nightly means. The peak at the frequency $16.916 \mathrm{~d}^{-1}$, marked as $f$, corresponds to the period $0.05912 \mathrm{~d}$. The group of peaks at the left-hand side of the diagram correspond to $1-\mathrm{d}, 0.5-\mathrm{d}$... aliases that also appear in the window function and to frequencies longer than the length of the observations in each night, which are probably the result of the noise, the detrending method used and the presence of nightly gradients

in a few individual nights. A consistent search for a second periodicity in the light curve with the various techniques discussed by Retter et al. (1997) yielded no positive results. We note that the power spectrum of the data after the subtraction of the linear trend from each night removes most low-frequency peaks, but the structure around the $17.916 \mathrm{~d}^{-1}$ frequency is quite similar to Fig. 3.

In Fig. 4 we present the mean light curve of the 1997 observations. The nightly trends were removed by subtracting a linear term from each night. The light curve shows a sinusoidal variation. The first harmonic fit to the data yielded a semi amplitude of $0.025 \pm 0.006$. The error corresponds to a $99 \%$ confidence level, and it was calculated by a sample of 1000 bootstrap simulations (Efron \& Tibshirani 1993). The best fit ephemeris for the periodicity is:

$$
\begin{aligned}
T_{\min }=\text { HJD } 2450612.397 & +0.05912 \mathrm{E} \\
\pm 0.002 \pm 0.00015 & (2.5 \sigma) .
\end{aligned}
$$

\section{Discussion}

The light curve of RW UMi in 1997 was modulated by the 1.4-h period. There are three obvious interpretations for this periodicity. It might represent the spin period of the white dwarf, a superhump variation or the orbital period of the binary system. In the following we examine the different models and try to classify the system in the light of the observations accumulated so far on the nova. 


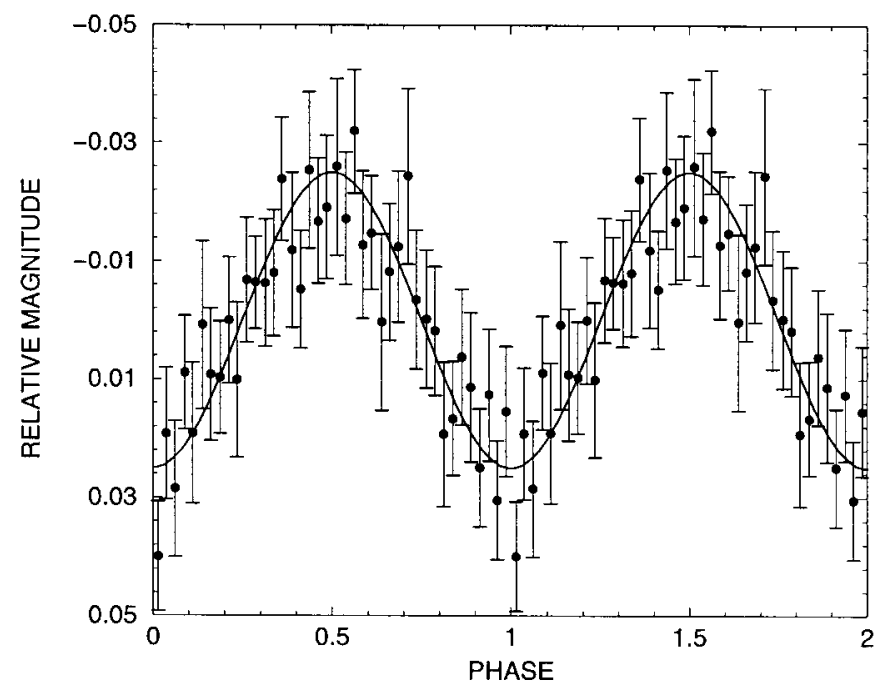

Fig. 4. The "clear" filter light curve of the nights in 1997 folded on the 1.4-h period and binned into 40 equal bins. The solid line represents the sinusoidal fit to the data. The bars show the standard error derived from the rms within each bin

\subsection{A spin period-?}

First, we discuss the question whether the system is an AM Her (polar) system (for a review see Warner 1995). The primary white dwarfs in these systems possess strong magnetic fields. Thus, the accreted matter from the companion star flows directly to the two poles of the rotating white dwarf whose spin period is synchronized with the orbital period of the binary system.

The spectra of RW UMi show a strong continuum and only weak emission lines. There are no strong high excitation lines, and the He II 4686 line is rather weak (Sect. 1). We thus believe that the polar scenario can be eliminated, since typical spectra of AM Her systems have a weak continuum and strong emission lines (Warner 1995). The typical optical spectra, radiated from accretion discs in postnovae, are characterized by a strong continuum, because the disc is usually optically thick in classical nova systems for at least a few decades after their eruption (Retter et al. 1999).

If the magnetic field is moderate the spin period is usually much shorter than the orbital period and an accretion disc might be formed around the white dwarf. These objects are termed "intermediate polars" (for reviews see Patterson 1994; Hellier 1996). Spin periods in intermediate polars range between $33 \mathrm{~s}$ in AE Aqr (Hellier 1996) to $1.44 \mathrm{~h}$ in Nova V1425 Aql 1995 (Retter et al. 1998). RW UMi might thus be an intermediate polar with the 1.4-h period being the rotation period of the white dwarf. The orbital period should then be somewhat longer than the spin period. The power spectrum of RW UMi (Fig. 3) does not show, however, other periodicities in addition to the 1.4-h period (Sect. 3). Therefore, it seems unlikely that the periodicity we have discovered is a spin period.

\subsection{A superhump period-?}

Superhumps are quasi-periodic oscillations that have been observed in the light curves of non-magnetic CVs with relatively short orbital periods (typically $P_{\text {orbital }}<4 \mathrm{~h}$ ). They either appear in the bright outbursts (superoutbursts) of the SU UMa subclass of dwarf novae or permanently in nova-likes. Superhump periods differ from binary periods by a few percent. They are understood as the beat periods of the orbital period and the apsidal (or the nodal) precession period of the accretion disc. For reviews on superhumps see Warner (1995) and Osaki (1996) and on permanent superhumps - Patterson (1999) and Retter \& Naylor (2000). If the 1.42-h period in RW UMi is a positive superhump, a superhump period excess of $1-4 \%$ is expected according to the observed values in superhump systems (Ritter \& Kolb 1998), leading to a binary period in the range $1.36-1.40 \mathrm{~h}$. If the periodicity is a negative superhump, the expected range in the binary period is $1.43-1.45 \mathrm{~h}$ since period deficits in negative superhumps are about half period excesses in positive superhumps (Patterson 1999). In both cases the orbital period is only a few percent different from the observed periodicity.

\subsection{The binary period-?}

Orbital periods of nova systems range between $1.425 \mathrm{~h}$ (GQ Mus) to $48 \mathrm{~h}$ (GK Per) with a peak around 3-4 h (Diaz \& Bruch 1997). The observed orbital period distribution of CVs presents a deficiency of systems between about $2 \mathrm{~h}$ and $3 \mathrm{~h}$, which is termed "the period gap" (Warner 1995). So far only three novae with established orbital periods below the gap have been discovered. This is compared with a few dozen novae with orbital periods above the gap (Sect. 1). If the 1.419-h periodicity observed in the light curve of RW UMi is its orbital period, it would be the shortest recorded binary period in a classical nova, although confirmation by radial velocity measurements is required. Table 1 presents the pre-nova and post-outburst magnitudes of the novae below the gap as well as several other properties. The data show that the brightness of the post-novae below the gap is still above the pre-nova state. RW UMi is much brighter than the progenitor value as well.

\subsection{RW UMi as a high galactic latitude nova}

RW UMi is a high galactic latitude nova and therefore probably a binary system in the halo of the galaxy. Halo $\mathrm{CVs}$ are believed to be relatively old systems compared with the population of CVs in the galactic disc (see e.g. Howell \& Szkody 1990). Thus, according to evolutionary theories halo systems should have shorter orbital periods (assuming they haven't bounced yet at the period minimum). Such a difference between the two populations has indeed been found (Howell \& Szkody 1990). The possibility that the orbital period of RW UMi is the shortest among all known novae is consistent with this idea. 
Table 2. Properties of the classical novae below the period gap

\begin{tabular}{|c|c|c|c|c|c|c|}
\hline Object name & year of outburst & orbital period & nature & pre-nova magnitude & post-nova magnitude & difference \\
\hline RW UMi & 1956 & $1.419-?^{1}$ & non-magnetic-? ${ }^{1}$ & $21^{2}$ & $18.8^{1}$ & 2.2 \\
\hline GQ Mus & 1983 & $1.425^{3,4}$ & magnetic-? ${ }^{3,4}$ & $22^{2}$ & $18.0-?^{4,5}$ & $4-?$ \\
\hline CP Pup & 1942 & $1.474^{6}$ & non-magnetic ${ }^{6}$ & $>17^{7}$ & $15.3^{6}$ & $>1.7$ \\
\hline V1974 Cyg & 1992 & $1.950^{8,9}$ & non-magnetic ${ }^{8,9}$ & $21^{8,10}$ & $16.5-?^{11}$ & $4.5-?$ \\
\hline
\end{tabular}

${ }^{1}$ This work; ${ }^{2}$ Duerbeck (1987); ${ }^{3}$ Diaz \& Steiner (1989); ${ }^{4}$ Diaz \& Steiner (1994); ${ }^{5}$ Diaz et al. (1995); ${ }^{6}$ Patterson \& Warner $(1998)$;

${ }^{7}$ Warner (1995); ${ }^{8}$ Retter \& Leibowitz (1998); ${ }^{9}$ Skillman et al. (1997); ${ }^{10}$ Pavelin et al. (1993); ${ }^{11}$ Goransky (2000).

\subsection{The mass accretion rate and thermal stability of $R W U M i$}

In this section we estimate the mass transfer rate in RW UMi to assess whether it has dwarf nova or nova-like characteristics. Permanent superhumpers (and nova-likes) are believed to occur in CVs with thermally stable discs, while dwarf novae (including SU UMa systems) are thermally unstable. Based on the thermal-tidal disc instability model (Osaki 1996), assuming that the disc is the dominant light source in the visual band, and neglecting a possible contribution from the hot white dwarf in post-novae, Retter \& Leibowitz (1998) and Retter \& Naylor (2000) developed a way to check the thermal stability state of disc-accreting CVs. In the following, we apply this method to RW UMi to obtain its mass transfer rate.

The visual magnitude of the post-nova is $m_{V} \approx 18.8$ and the distance $-4200-5840 \mathrm{pc}$ (Sect. 1). The interstellar reddening is $A_{V}=0.1$ (Anhert 1963; Cohen 1985). For the mass of the white dwarf, we take $M_{\mathrm{WD}}=0.7 M_{\odot}-$ typical of CVs below the gap (Patterson 1998). The absence of eclipses (Sect. 3) limits the inclination angle to $i=0-65^{\circ}$. Inserting all these values into Eq. (3) of Retter \& Naylor (2000), we obtain $\dot{M}=1.2-8.610^{17} \mathrm{~g} \mathrm{~s}^{-1}$. The critical mass transfer rate, calculated by Eq. (2) of Retter \& Leibowitz (1998), assuming that the orbital period is $1.4 \mathrm{~h}$ (Sect. 4.3 ) is $0.510^{17} \mathrm{~g} \mathrm{~s}^{-1}$. The system is thus very likely to be thermally stable. This fact together with the long-term light curve of the nova (Fig. 1) that does not show dwarf nova outbursts (although obviously outbursts could have been missed) seem to reject the possibility that the post-nova is a dwarf nova system, and therefore it should be a nova-like system. A similar test applied to the progenitor of the nova at $m_{V}=21.0$ (Sect. 1 ) yields $\dot{M}=0.2-1.110^{17} \mathrm{~g} \mathrm{~s}^{-1}$. This range straddles the limiting value, so the thermal stability state of the pre-nova cannot be determined.

\subsection{Final classification of the system}

A definite classification of the system cannot be made. An AM Her model doesn't fit the nova properties (Sect. 4.1). An intermediate polar model might be applicable to RW UMi, but only if an additional short-term periodicity (the spin period) or a relatively long period (which would be the orbital period, making the 1.4 -h period the spin period) is found in the future. We note that it is very likely that an accretion disc is formed around the white dwarf in RW UMi as the AM Her scenario has been rejected and since it is believed that most intermediate polars have accretion discs (Patterson 1994).

A dwarf nova (SU UMa) model seems to be ruled out by the long-term behaviour of the light curve in 1995-1999 (Fig. 1) that does not show any dwarf nova outburst, and by our findings that the system is almost certainly thermally stable (previous section). RW UMi is therefore most likely a system with a short orbital period in a high state. As such, it should have permanent superhumps according to Osaki (1996), however, the observations accumulated so far cannot confirm this phenomenon, probably because the nova is relatively faint.

\section{Summary}

A photometric period of 1.4-h was detected in the light curve of the classical nova RW UMi. We argue that it is most likely the orbital period of the system or a superhump period. In both models Nova Ursa Minoris 1956 has a very short binary period. If confirmed, RW UMi will be then the fourth nova below the gap and the third to be non-magnetic.

Acknowledgements. We thank the referee, Daisaku Nogami, for many valuable remarks. We are grateful to Hasan Esenoglu who re-raised our interest in this object. Tim Naylor is acknowledged for a careful reading of the manuscript and for many useful comments. We also thank Coel Hellier and Sandi M. Catalan for several fruitful discussions, and John Dan and the Wise Observatory staff for their expert assistance with the observations. AR is supported by PPARC. Astronomy at the Wise Observatory is supported by grants from the Israeli Academy of Sciences.

\section{References}

Anhert, P. 1963, IBVS, 19

Cohen, J. G. 1985, ApJ, 292, 90

Diaz, M. P., \& Bruch, A. 1997, A\&A, 322, 807

Diaz, M. P., \& Steiner, J. E. 1989, ApJ, 339, L41

Diaz, M. P., \& Steiner, J. E. 1994, ApJ, 425, 252

Diaz, M. P., Williams, R. E., Phillips, M. M., \& Hamuy, M. 1995, MNRAS, 277, 959 
Downes, R. A., \& Duerbeck, H. W. 2000, AJ, in press

Duerbeck, H. W. 1987, Space Sci. Rev., 45, 1

Efron, B., \& Tibshirani, R. J. 1993, An Introduction to the Bootstrap (Chapman \& Hall)

Esenoglu, H. H., Saygac, A. T., Bianchini, A., et al. 2000, A\&A, in press

Goransky, V. P. 2000, vsnet-obs 26924, http://www.kusastro.kyoto-u.ac.jp/vsnet/ Mail/obs26000/msg00924.html

Hellier, C. 1996, in Proc. IAU Colloq. 158, Cataclysmic variables and related objects, ed. N. Evans, \& J. H. Wood, (Kluwer, Dordrecht), 143

Howell, S. B. 1988, IAUC, 4672

Howell, S. B., \& Szkody, P. 1990, ApJ, 356, 623

Howell, S. B., Szkody, P., Kreidel, T. J., \& Dobrzycka, D. 1991, PASP, 103, 300

Kaluzny, J., \& Chlebowski, T. 1989, Acta Astron., 39, 35

Kukarkin, B. V. 1962, IBVS, 18

Netzer, H., Heller, A., Loinger, F., et al. 1996, MNRAS, 279, 429

Osaki, Y. 1996, PASP, 108, 39

Patterson, J. 1994, PASP, 106, 697

Patterson, J. 1998, PASP, 110, 1132

Patterson, J. 1999, in Disk Instabilities in Close Binary Systems, ed. S. Mineshige, \& C. Wheeler (Universal Academy Press, Tokyo, Japan), 61

Patterson, J., \& Warner, B. 1998, PASP, 110, 1026
Pavelin, P. E., Davis, R. J., Morrison, L. V., Bode, M. F., \& Ivison, R. J. 1993, Nat, 363, 424

Retter, A. 1999, IAUC, 7263

Retter, A., \& Leibowitz, E. M. 1998, MNRAS, 297, L37

Retter, A., \& Naylor, T. 2000, MNRAS, in press

Retter, A., Leibowitz, E. M., \& Ofek, E. O. 1997, MNRAS, 283,745

Retter, A., Leibowitz, E. M., \& Kovo-Kariti, O. 1998, MNRAS, 293, 145

Retter, A., Naylor, T., \& Leibowitz, E. M. 1999, in Disk Instabilities in Close Binary Systems, ed. S. Mineshige, \& C. Wheeler (Universal Academy Press), 91

Ringwald, F. A., Naylor, T., \& Mukai, K. 1996, MNRAS, 281, 192

Ritter, H., \& Kolb, U. 1998, A\&AS, 129, 83

Shafter, A. W., \& Campbell, R. 1990, BAAS, 22, 1211

Skillman, D. R., Harvey, D., Patterson, J., \& Vanmunster, T. 1997, PASP, 109, 114

Slavin, A. J., O'brien, T. J., \& Dunlop, J. S. 1995, MNRAS, 276, 353

Stetson, P.B. 1987, PASP, 99, 191

Szkody, P. 1994, AJ, 108, 639

Szkody, P., \& Howell, S. B. 1992, ApJS, 78, 537

Szkody, P., Howell, S. B., Mateo, M., \& Kreidel, T. J. 1989, PASP, 101, 899

Warner, B. 1995, Cataclysmic Variables Stars (Cambridge University Press) 